\title{
A Home-Based Care Model for Outpatient Cardiac Rehabilitation Based on Mobile Technologies
}

\author{
Antti Särelä \\ The Australian E-Health Research Centre \\ CSIRO \\ Brisbane, Australia \\ Ilkka Korhonen \\ ICT for Health \\ VTT \\ Tampere, Finland
}

\author{
Jukka Salminen, Esa Koskinen, Ole Kirkeby \\ Nokia Research Center \\ Nokia Oyj \\ Helsinki, Finland
}

Darren Walters

The Prince Charles Hospital

Queensland Health

Brisbane, Australia

\begin{abstract}
Cardiac rehabilitation programs offer effective means to prevent recurrence of a cardiac event. However, poor uptake of current programs have been reported globally. Home based model is considered as an alternative to avoid barriers related to hospital based programs. The challenge in a home based program is to re-establish the link between the patient and clinician regardless of their location. Mobile phones and internet technologies can potentially be used to enable a comprehensive home based care model. We developed tools and integrated model for home based cardiac rehabilitation based on a mobile phone with integrated accelerometer to measure physical exercise and diary to collect information on physiological risk factors and other health information. Video and teleconferencing are used for mentoring sessions and educational multimedia content is transferred via messaging systems to the patients phone to be viewed on demand. The model can be extended to management of other chronic conditions.
\end{abstract}

Keywords - cardiac rehabilitation, mobile technology, personal health systems, phone consultation, wellness diary.

\section{INTRODUCTION}

Cardiovascular disease (CVD) is the most common cause of death in Australia, accounting for $34 \%$ of all deaths in Australia in 2006 [1]. Cardiovascular disease including diseases of the heart, blood vessels and stroke, kills one Australian nearly every 10 minutes. This represents the single largest burden of any disease. CVD is also a major driver of cost to the community in terms of health care expenditure. More than $\$ 5.5$ billion is spent annually on the acute and chronic management of these conditions. Ischemic heart disease is the leading cause of mortality and accounts for $19.2 \%$ of all deaths in both males and females. The total burden of this disease is likely to increase given the increase in morbid obesity and diabetes and the growing number of elderly patients in whom these diseases are more common.

A number of modifiable environmental and patient specific factors increase the chance of developing coronary heart disease. These include smoking, high blood cholesterol, physical inactivity, diabetes, high blood pressure, obesity and depression, social isolation and lack of social support. Cardiac rehabilitation has provided an avenue for reducing cardiovascular risk in patients into the future by positively influencing these factors. A comprehensive cardiac rehabilitation program offers a long-term strategy incorporating medical evaluation, 'prescribed' exercise, cardiac risk factor modification, education and counseling [2]. Patients with cardiac disease are encouraged and supported to achieve and maintain optimal physical and psychosocial health through the development of a therapeutic partnership with a multidisciplinary team of health professionals. The eventual goal of the program is to engage patients with permanent lifestyle change regarding their health behaviors, i.e. behavioral change. There is good evidence for the effectiveness of cardiac rehabilitation particularly from studies of patients with myocardial infarction and following coronary revascularization.

Unfortunately low levels of patient participation (14-43\% after myocardial infarction) are consistently reported in Australia, France, the UK, New Zealand and the USA [3] [4]. These low patient participation rates are reported to be a consequence of low levels of service provision, referral and invitation, and of poor uptake by patients. Patients with the best rates of participation in cardiac rehabilitation programs have tended to be male, middle-aged and diagnosed with uncomplicated myocardial infarction. Those most likely to benefit such as those with significant functional impairment, older patients, women and ethic groups are the least likely to participate in a program. However a number of other very practical factors such as difficulties with transportation, lack of interest, dislike of classes/hospitals, work or domestic commitments, rural residence, location and accessibility and car parking all influence utilization of cardiac rehabilitation [5].

Improvement in rates of utilization of cardiac rehabilitation should be investigated. As lifestyle issues may act as significant barriers to uptake and adherence, a range of different models for rehabilitation program offerings should be available and selectable for the patients, according to their own preferences and needs. These include models for home based 
rehabilitation programs, which could benefit from use of innovative electronic and internet based technologies in program delivery and patient empowerment and overcome the limitations and barriers to adherence that plague traditional systems. Home-based programs have been proposed as an alternative model and shown to be a viable option for cardiac rehabilitation in some studies. One of first home-based programs was called MULTIFIT that focused on comprehensive risk factor reduction including for example computer based nutritional counseling [6]. The Heart Manual program, which is a home based rehabilitation model using counseling by specially trained Mentors, is in a routine use in UK [7][8]. One potential benefit for the home-based rehabilitation, in addition to increased access to the program, may be better long-term adherence to life-style changes as the changes in exercise patterns would be implemented immediately in the every-day settings as contrast to institutional settings which are typical in traditional programs [9].

Mobile phone provides an especially attractive media to support health and chronic disease management programs administered over distance. As a technology platform, it integrates sufficient computing power, user interface, memory, and communication capabilities to run applications needed for personal health management. From individual user's perspective it is considered as a sufficiently personal and trusted device to store personal messages and carry out payments and other transactions of monetary value, and is available while making daily health related behavioural choices. Furthermore, mobile phone is regularly carried on even while exercising or doing some other daily tasks. According to an earlier study, approximately $90 \%$ of working age users kept their mobile phones along while exercising due to availability or safety reasons [10]. Mobile phones are attractive tools from health service providers' perspective, too, because they have very high penetration rate in most countries (e.g. in Australia 99\% and Finland exceeding 100\%), and in many countries significantly higher than for example broadband internet penetration. Hence, a mobile phone is also potentially a non-discriminating service media. Some earlier studies have successfully used mobile phone applications to wellness management [11][12][12]

We developed a home-based service model for cardiac rehabilitation based on mobile technologies. The driving principle of the model is to efficiently utilise mobile phone as a means to deliver service to outpatients participating safely in cardiac rehabilitation in their own homes or wherever they want. The mobile phone platform is utilised both in health and exercise monitoring, delivery of motivational and educational materials and messages to the patients, for sharing follow-up information with a case Mentor, and for performing weekly phone consultations and goal setting during the program. In addition, a special web-service was built to allow rich content and more elaborate personalised feedback to support those users who have also access to internet. We hypothesize that this model offers a cost-efficient alternative for cardiac rehabilitation.

We have organised this paper as follows. Design section defines the high level system requirements and describes the main software components used in the project. Implementation section explains the integrated system setup, home-based care model and how the technologies will be used in the care process. We describe the status and main findings in Results section followed by discussion and future directions of the work.

\section{DESIGN AND ENABLING TECHNOLOGIES}

\section{A. Requirements}

The goal of the design process was to develop a homebased technology enabled care model according to the national framework for cardiac rehabilitation processes and principles [14].

As a starting point for the design of the new model, we used the current centre-based program at Queensland Health and other generally applied home-based rehabilitation programs such the Heart Manual [8] as a starting point for the design of the new model. The current rehabilitation program targets primarily at improving patient's physical activity levels through participation in a 6 week intervention program, and is traditionally implemented in the form of group meetings and guided exercise sessions twice a week. In addition, the program includes health behavioral counseling on diet, stress management, risk factor education etc. The primary focus of the program is on physical exercise, and the fact that brisk walking is the most commonly recommended exercise for the target population, selection of the technology tools was accordingly chosen to optimize for the monitoring of exercising, especially walking. The rehabilitation goals and content of the program were kept unmodified for our homebased program.

The requirements for the technology platform were derived by investigating the current care practices and most recent research on both technology and clinical care models. The model was developed in collaboration with a multidisciplinary team of clinicians working on cardiac care and rehabilitation within Queensland Health. The high level design requirements for the technology were, therefore, defined as:

- Easy transferability to remote and rural sites, with minimal or no technology installation and set-up at patient's home, as a prerequisite.

- Use of cost efficient technologies ensuring possibilities for large scale roll out should be preferred.

- As the primary focus of the program is on exercise, and especially walking, the logging, monitoring and receiving feedback on walking and stepping activities should be as easy as possible, preferably automatic.

- Other areas of health behavioral counseling should be supported (weight management, stress management, blood pressure and cholesterol monitoring, diet management).

- Sharing of information content and motivational material in electronic form should be cost-efficient and easy.

- Flawless communication between the Mentor and the patient should be made easy. 
- The technologies should support patient empowerment and augment their own possibilities to follow up on selfprogress and management of their health.

Furthermore, it was emphasized that the model is not meant to replace traditional group-based face-to-face rehabilitation programs but to offer an alternative, potentially more favorable model to some patients, who are eligible for home-based rehabilitation. In other words, it does not need to fit for all patients but must critically meet the needs of a significant subgroup of them. It is one of the challenges of the planned trials to exactly identify the optimal eligibility criteria for this model.

\section{B. Mobile Phone Tools: Step Counter and Wellness Diary}

Step Counter (SC) is a mobile tool that records a user's movements (steps) automatically. Since most people have their mobile phone with them wherever they go, an accelerometer inside the phone is practical for monitoring a user's physical activity during the day. SC is available as a free download from Nokia Beta Labs [15][16]. The SC counts the user's steps, estimates the energy expenditure, distance, and intensity. Great care has been taken to ensure that the step count is accurate regardless of how the user carries the phone around, and in addition, the algorithm is designed so that it can reject false steps, for example picked up while driving. The $\mathrm{SC}$ allows a scheduler to start and stop the step counting at pre-defined times every day in order to save battery power.

Wellness Diary (WD) is a mobile tool for collecting, storing, and visualizing a wide variety of wellness-related parameters [17] [18][19].It is also available as a free download from Nokia Beta Labs [15], and it runs on most mobile phones that come with the Symbian S60 operating system. Currently WD supports entry of parameters such as weight, exercise, steps, eating, stress level, working hours, sleeping hours, blood pressure, fat percentage, blood sugar, waist circumference, and tobacco and alcohol consumption. The user can select the most suitable parameter subset, and personalize the main view of the application accordingly. Images taken with the phone's built-in camera can be associated with a health entry such as eating.
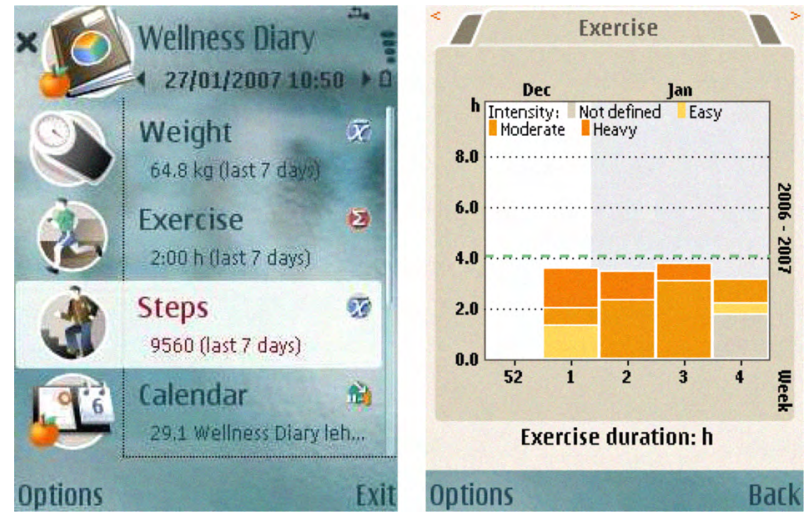

Figure 1.

Screenshots of Wellness Diary: the main view (left) and the duration and intensity of exercise (right) panel.

WD supports wireless data transfer over Bluetooth connectivity of blood pressure meter and weighting scales from AND-Medical. The implementation is based on the proprietary protocol of AND-Medical. When the WD starts, it imports summary data from the SC and SportsTracker [20], applications without the need for any user input. The Bluetooth connectivity feature for the weight scale and blood pressure as well as the SportsTracker software are not however used in the final integrated system for the home based cardiac rehabilitation model described in the section III.

The data repository of WD is a semi-structured, tree shaped data model, which is mapped to a generic relational model on the top of SQLite relational database. The application supports a fully featured two-way synchronization with Wellness Diary Connected (WDC) service, including the media items. The synchronization is based on SyncML. Its data model is close to the service side making it a client side Personal Health Record (PHR) and a mobile hub as well.

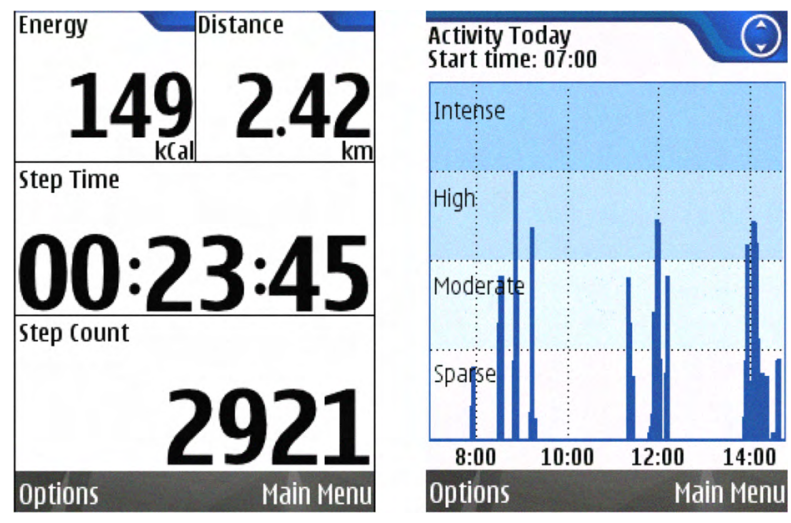

Figure 2. Screenshots of Step Counter : the main view (left) and the step frequency (min-by-min) over a short period of a day. Different time windows may be chosen for the feedback and review of the data.

\section{Wellness Diary Connected platform}

Wellness Diary Connected (WDC) is a web service for recording PHR and supports largely the same parameters as those in the WD mobile application. It consists of three parts:

1) End User View

End User View consists of several views such as a customizable dashboard (main view), charts, entry list, goal settings, calendar, images and messaging.

The dashboard (Fig 3.) provides a single view to all user data, consisting of plug-ins that can be freely selected, dragged, and dropped. The system stores the user settings. The user can select the items, like charts, summaries, feedback, messages and goals she/he would like to follow.

User research studies have shown that the perceived value of the WD mobile application is relatively modest in the long term since the charts generated and the feedback given are not sufficiently valuable to justify the effort required to keep inputting health data manually. Therefore, in the case of the WDC service, a deliberate attempt has been made to provide meaningful feedback that is easy for the user to understand. The four central concepts used in the feedback and motivation of the users are traffic light indicators, a simple measure of physical activity, a goal setting and a verbal feed back. 


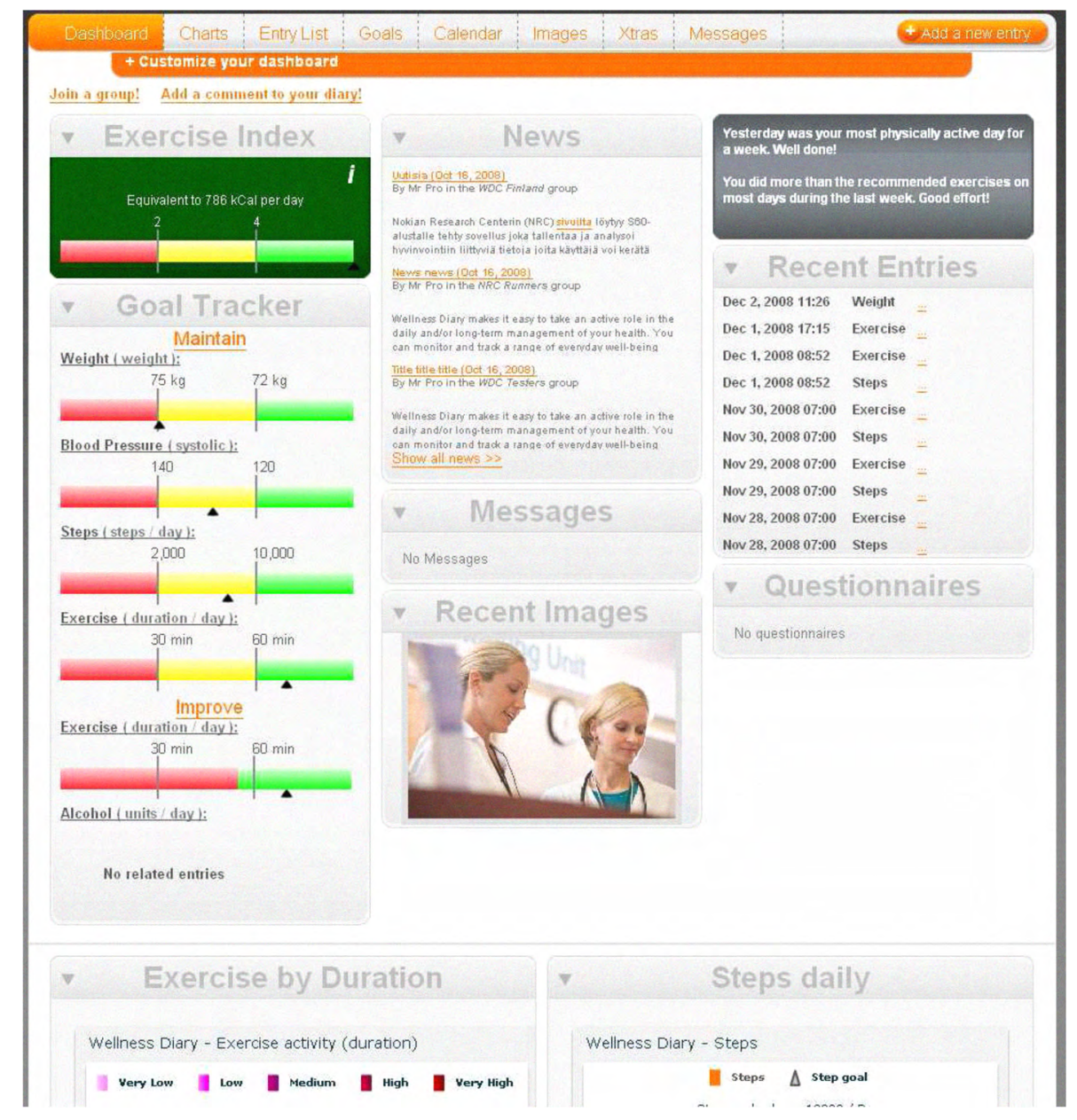

Figure 3. Screenshot of Wellness Diary Connected: the dashboard view.

Traffic light indicators are often used when assessing whether a person has a certain health risk, for example, resulting from too much smoking or not having enough exercise. The labeling is very intuitive, with green (all is well), yellow (reason for concern), and red (action should be taken). As a second level of information, it can be helpful for the user to know how far away the current rating is from neighboring ratings. For example, a diastolic blood pressure of 92 is too high and so it merits a yellow traffic light but it can be argued that it is closer to green than red. A point on a colored bar divided into three equally large areas (green, yellow, and red) immediately shows the current rating relative to the others.

A simple measure of physical activity is crucial in order to make users aware how much exercise they are supposed to get. The traditional measure of Calories has the disadvantage that it depends on the user's weight, and most people are unaware exactly what it means. A more convenient unit is Metabolic Equivalent (MET), which is the caloric consumption of a person while at complete rest. The Wellness Diary Connected service uses so called Exercise Units to indicate the energy consumption. The Exercise Units are based on the MET-hours, which are calculated by multiplying the MET rating by the duration of the workout. Walking at a moderate pace is equivalent to approximately four METs, cycling $20 \mathrm{~km} / \mathrm{h}$ is seven METs, and running $10 \mathrm{~km} / \mathrm{h}$ is 10 METs. Data imported from the SC are automatically converted to MET-hours by the service. The minimum recommended amount of daily physical activity [21] averaged over one week, can be summarized as two MET-hours at moderate intensity (MET between three and six), and another two MET-hours of vigorous exercise (MET greater than six). Consequently, it is sensible to use a traffic light indicator, which classifies more than four MET-hours daily as green and less than two MET-hours as red.

Goal setting is very important for the motivation of the user. There are two types of goals, described as 'Maintain' and 'Improve'. 'Maintain' is for keeping a health parameter inside a certain range. Perhaps someone decides that ideally, their weight should be below 80 kilos but they would be willing to accept that it occasionally is a little higher, as long as it does not go above 83 kilos. The traffic light indication would then be green for an entry below 80 kilos, yellow for an entry between 80 and 83 kilos, and red for an entry above 83 kilos. 
'Improve' is a time-based goal so that the user specifies not only the goal but also a target date for when it should be achieved. As opposed to the 'Maintain' mode, there is no yellow traffic light indication in the 'Improve' mode, only red and green. The user is either ahead of schedule or behind schedule. As a second level of information, the status can be indicated by a point on a bar, which is green at one end and red at the other end. The red part of the bar expands with time so that on the target date, only an entry that satisfies the goal will produce a green traffic light.

\section{2) Professional View (ProView)}

Professional View (ProView) is a separate interface for professional users. The functionalities are aiming at user management, intervention support, and research support.

ProView allows managing groups, patient monitoring and analysis, news postings, and a system wide messaging with attachments. It also allows to the professionals to make search on users, create questionnaires, and access the users' data.

\section{3) Administrator View (AdminView)}

Administrator View (AdminView) is meant for system administrators and it allows creating and managing user groups and providing Admin, Proview rights.

One of the design criteria of the WDC was to make the system customizable by the end user as well as easily extendable by system designers. The data model was designed to be extendable from the beginning and new simple charts to be easily added to the system. Currently there are about 15 health parameters supported. Charts use system's generic XML API to retrieve and aggregate data. On the client side hand WD mobile client uses dynamic data model and enables for instance data synchronization of new parameters without any modifications to the application itself [17].

The web service part uses Spring application framework and it's model-view-controller architectural pattern to layer. For synchronization Funambol open source SyncML server has been used. The platform also supports the OpenID single-sign on standard for the user authentication [22].

Figure 4. depicts the overall system architecture and mentions some of the used technologies.

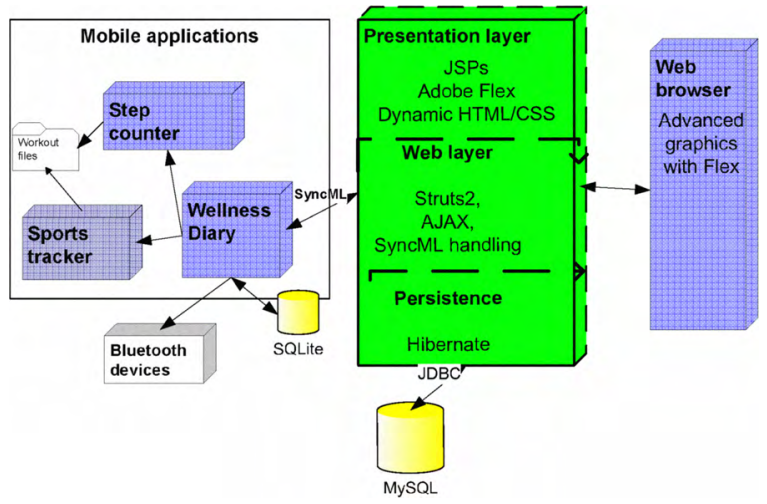

Figure 4

The overall system architecture and selected technologies.

\section{TECHNOLOGY INTEGRATION}

\section{A. General system description}

The Care Assessment Platform (CAP) is a project and system to create a new model of home-care for cardiac rehabilitation. The CAP system diagram is presented in Fig. 5. The personal mobile phone is the main technology component in the system and will be used for three purposes:

- To provide mentoring and education to the patient via video or normal telephone calls and using other messaging tools.

- To measure and collect physiological data, health and behavioral information from the patient by using the WD and SC.

- To provide patient feedback from the self observations and measurements through WD, and to listen to relaxation audio files (by using the standard Music Player of the device) to support patient in self-management

In our model of home-based cardiac rehabilitation program, patients receive both the mobile tools and access to WDC service. However, they can carry out all the main tasks by using the mobile phone only, and WDC may be used as desired. The WDC is used by the Mentor (who is associated for each patient as a health coach) as a means for monitoring the progress of the patients in their program and hence to guide the phone guidance. The SC is installed on the patient's mobile phone and will launch automatically each morning to register any steps taken during the day - all that is needed by the patient is to carry the phone along during the day. The WD is also installed on the patient's mobile phone, and allows registering (by typing in manually) other relevant parameters such as their weight, blood pressure, stress and sleep, and consequently getting feedback on their condition either through the application itself or through the Mentor (phone counselling). The same phone will also be used for receiving the motivational and educational materials (SMS, Multimedia) as well as for phone counselling.

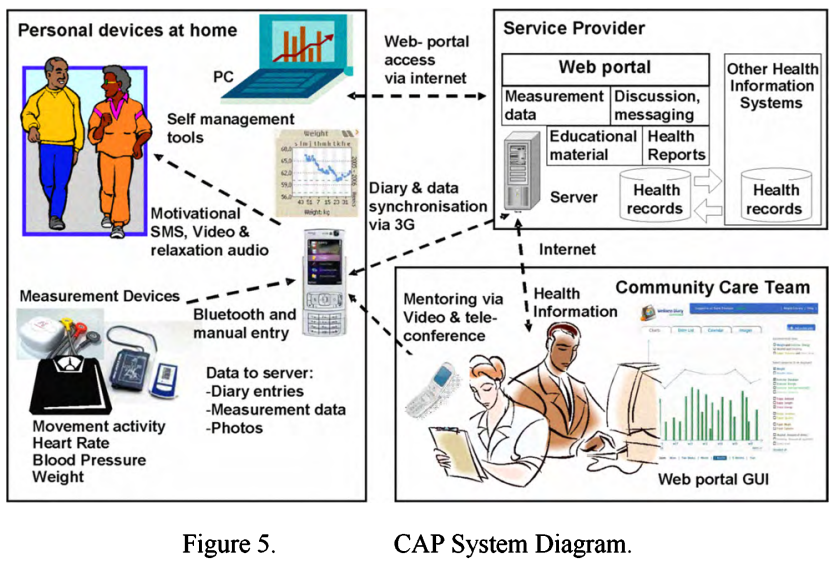

Patients use separate personal devices to measure their weight and blood pressure. The mobile phone measures patient's movement activity with the SC software. We 
evaluated the SC against a standard pedometer (Omron Walking Style) and found the measured step counts to be comparable in 4 different walking activities of 7 subjects. The measured mean difference between the SC and Omron was $4.5 \%$ in step counts during uphill walking. The difference was smaller in all the other measured walking activities. The patient is instructed to carry the mobile phone with him/her throughout the day and especially while exercising, in which case the exercise will be automatically captured by SC and transferred to WD. The WD variables that the patient is instructed to enter manually on the phone are: Weight, Fat \%, Blood Pressure, Exercise (other than walking), Eating, Sleep, Stress and tiredness, Alcohol and Smoking. Only exercise that can not be measured with the SC such as swimming will be entered manually. The diet information can be collected via the WD (rough estimation on the healthiness of the food) and additionally by taking photos of the meals by using the phone's digital camera to support Mentoring. All the measured and manually entered data is transferred from the phone to a remote server running WDC web service. The web-portal provides access to the collected patient information for the Mentors as well as for the patients, if they desire to do so.

The data synchronization requires currently a $3 \mathrm{G}$, GPRS or WLAN connectivity, which are all supported by the mobile terminals Nokia N96 and N95, used in the CAP project. The phones include a $3 \mathrm{G}$ phone plan that is used for all communication and data transfer by default. The user can also manually select the preferred connection for accessing the Internet and synchronizing the Wellness Diary with the service. WLAN is a free or a low cost connection method, whenever there is an access point available e.g. at the user's home. When the user is on the move then the $3 \mathrm{G}$ or GPRS networks can be used instead.

\section{B. Cardiac Rehabilitation Program Description}

As mentioned above, our rehabilitation program structure was developed according to the Australian national guidelines [14] [21] to cover all the core components of a comprehensive cardiac rehabilitation program such as patient assessment, lifestyle risk factor management, biomedical risk factors, and psychosocial issues. The process is briefly explained below.

When the patient is referred to a program he/she will be contacted by personnel and enrolled through an initial assessment appointment at the hospital or community care centre. The appointment takes a few hours and includes baseline measurements, training to use the different technologies and software, education on avoiding risks, correct exercise levels, and briefing on details such as contact persons. Most importantly, during this first appointment the patient meets his/her personal Mentor who will assist in setting the personal exercise and lifestyle goals for the first week in the home program.

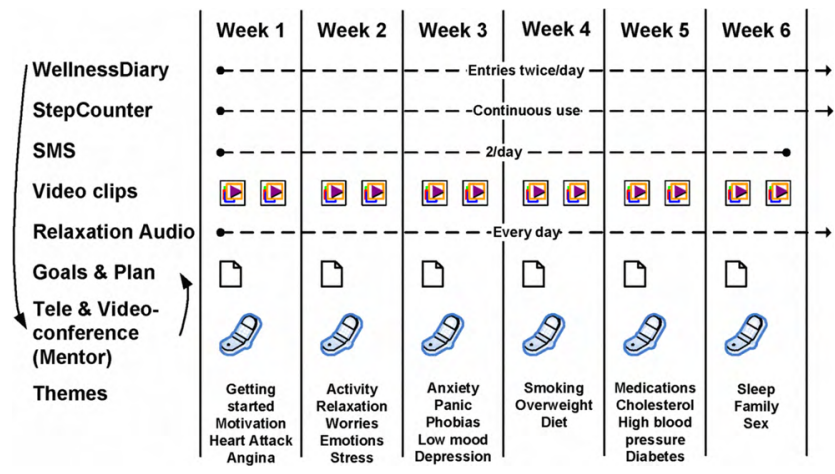

Figure 6. Home Program Overview.

The home program covers 6 weeks (Fig. 6), which are divided in themes that focus on different lifestyle aspects, risk factor management and education on the disease. The main intervention is provided via weekly video or tele-conference sessions with the patient and his/her Mentor by using mobile phone. The Mentor has access to patient data from WDC, and the patient's progress in comparison to the set goals will be discussed. The Mentor will provide guidance to the patient in setting the new goals for the following week. The patient writes down his/her own goals on exercise and behavioral modifications every week. The Mentoring sessions are appended with SMS messages that contain links to educational multimedia material (video clips) and short motivational messages on the topic of the weekly theme. The phone includes relaxation audio files that the patient can listen to any time and anywhere. The educational multimedia material, relaxation audio and messaging services are provided by Multi-ed Medical, Australia, Pty Ltd [23].

The daily program at home consists of light to moderate levels of physical exercise. Walking is the main recommended activity as it is not too strenuous and most patients are comfortable with walking as a mode of exercise. The patients will initially start walking at a low intensity level and increase the intensity and duration gradually through the program. The goal is to engage the patient in at least 30 minutes of moderate level of physical activity on most of the days of the week in the end of the program [21].

The patient uses the WD and SC on a daily basis as described above for monitoring activity and other health data. The SC synchronised with the WDC service is a tool for the Mentor to measure patients' walking activity and to assess their adherence to the guidelines. The patients can also use SC and WD as tools to observe their own progress against the personal goals set into the system with the Mentor.

The active phase of the rehabilitation program ends after 6 weeks. The patient travels second time to the community centre for post program assessment where the measurements at the initial assessment phase will be repeated. The patient's progress and current status is evaluated and a continuation plan for the next few months is designed with the Mentor. The mentoring sessions over the video and telephone calls will not be continued, but the patient can still continue using the technology tools for self management. 


\section{RESULTS}

The WDC platform has been installed and set-up at The Australian E-Health Research Centre, CSIRO, and is currently under acceptance testing before a trial within an outpatient cardiac rehabilitation program. The main challenges related to the use of mobile technologies that have been encountered during the system implementation phase are the following:

- The health care provider might prefer the WDC service to be installed within their IT infrastructure as it contains patient data. The service model, however, requires that the patients also should have access to their own data via the phone or internet. This and the data synchronization process between the WD and WDC service require opening the health care providers firewall for outside internet traffic. The changes in the IT infrastructure involving data security issues are usually tightly regulated within a health care service, which have to be accounted for in the system setup phase.

- The patient might not have a phone plan that would support all the services, which currently require a GPRS, 3G or WLAN connectivity. Additionally the costs of data transfer should not be on the patient's expense. A simple solution for the health care provider is to provide an adequate $3 \mathrm{G}$ plan and a new SIM card to the patient. However, the patient should be responsible for his/her personal phone use. Our solution is to provide a phone plan where the bill can be split between business and personal use. This will however require the patient to sign a contract with the specific tele-operator and possibly upgrade his/her phone plan and change the phone number.

- The system features require that the patient starts using a new phone model that has for example video call capabilities. This introduces training requirements and risks in user acceptance. Ideally the patient would use only one phone model and plan for all the services ranging from health services to personal entertainment.

The platform will be validated in a Randomized Controlled Trial (RCT) involving cardiac rehabilitation programs conducted in The Prince Charles hospital in Brisbane as well as Caboolture and Redcliffe hospitals of Queensland Health services. The trial is jointly funded by CSIRO and Queensland Health, Australia. The goal is to provide evidence that the technology enabled and home-based care model is a cost effective and viable alternative for cardiac rehabilitation in terms of health outcomes. Positive results would imply potential for large scale roll out as an alternative care model. The ethical committee has approved the project plan and the patient recruitment will commence in the first quarter of 2009. The trial diagram is presented in Fig 7. After consent the patients will be randomized into two study arms. The "IT Group" will be using the mobile phone technologies and receive rehabilitation in a home environment. The "No IT Group" will be attending a traditional hospital based rehabilitation program. By comparing the results from both groups it is possible to evaluate the feasibility of the proposed model. The main outcome measure is the adherence to exercise guidelines, which will be measured with a physical activity questionnaire and a reference pedometer used in both IT and No IT groups. Other outcome measures include risk factors such as blood pressure and lipids, patient satisfaction, quality of life, diet status, medication adherence, completion rate, readmissions, and costs. These will be measured during the pre- and post assessment with questionnaires, standard clinical tests and measurements, and including data from the patients' health records. The results of the trial will be available in late 2009.

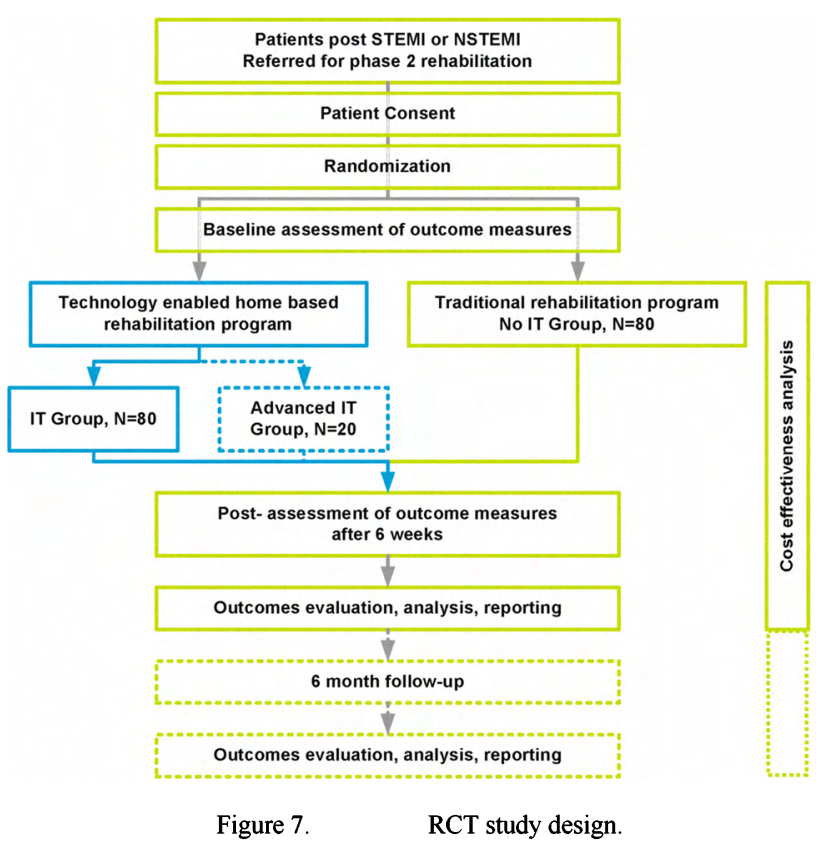

\section{DISCUSSION AND FUTURE DIRECTIONS}

We have developed a home-based care model for outpatient cardiac rehabilitation based on mobile technologies. The model includes re-design of the rehabilitation process to efficiently utilize available technologies, as well as the technology platform to support the implementation of the care model in the community. The strengths of the model include its true mobility and transportability to allow for an easy implementation at various geographical settings with minimal logistic or set-up requirements. The utility, validity and costefficiency of the model will be studied in a randomized controlled trial during 2009. This study will also demonstrate the ability of the relatively aged cardiac patients to cope with the given technologies in real life settings.

The presented platform offers an affordable and highly scalable solution for home based chronic care models. The model is now designed for cardiac rehabilitation but could be extended to support also other chronic diseases by some modifications to the content and monitored variables. The main benefit in using mobile phones and web services is in the affordability and wide spread use of these technologies.

The mobile phones are developing rapidly and it is likely that the essential features will be standard in the future midrange phone models further reducing the overall setup costs. 
The main operating costs for the health care provider will be the phone calls from by the Mentor to the patients, which is likely to be less expensive than the face-to-face consultations requiring the added cost of travelling time. The data transfer from the patient's phone to the server is limited to few hundred kiloBytes per day which is a small operating cost per patient.

The system would greatly benefit from standard and open interfaces to transfer data from a range of third party measurement devices to the mobile phone. Currently the device manufacturers are either using proprietary radios that are not compatible with mobile phones at all or proprietary Bluetooth protocols, making it necessary to develop individual interfacing software for each device. The most common wireless interface in the mobile phone is Bluetooth that unfortunately requires significant battery power making it unpractical for small battery operated wearable health monitoring devices that would not need the high data transfer capacity offered by the Bluetooth. The future Bluetooth Low Energy is focused on use cases, which are not covered well with the standard Bluetooth solution. It is an open short range radio technology offering connectivity between mobile devices and small, button cell battery -powered wearable devices such as watches, sensors and gadgets [24]. The activities by the multi-organizational collaborative body, the Continua Alliance [12] are expected to enable interoperable e-Health ecosystem in the future. We will trial a Bluetooth enabled Heart Rate and movement activity monitor in the Advanced IT Group of the trial (Fig. 7). The goal is to provide richer measurement data during the patients' home exercise to better assess the heart function, physiological status and development during the rehabilitation.

The web service in the future may integrate with external system components offering advanced decision support features. One of these is an Evidence Based Medicine Decision Support system and technology (EBMeDS) developed by Finnish medical society (Duodecim, Helsinki, Finland). Originally EBMeDS was designed as a professional tool for supporting clinical work but its rule system can be extended to wellbeing area and to provide feedback to end-users and patients.

In summary, mobile phone technologies and related web services are potentially enabling tools for home-based care models that require tools and services for interventions through both mentoring and self management to facilitate sustainable behavioral modifications. Our model for cardiac rehabilitation at home could be extended to other chronic illnesses in the future. The on-going clinical trial of the CAP project will provide outcome data on the benefits of the model.

\section{ACKNOWLEDGMENT}

We would like to acknowledge all the CAP project team at Queensland Health, Northside Primary and Community Health Services,at the Caboolture, Redcliffe and The Prince Charles Hospitals for their continuous support and invaluable clinical expertise.

\section{REFERENCES}

[1] National Heart Foundation of Australia (Report by Vos T and Begg S, Centre for Burden of Disease and Cost-effectiveness, University of
Queensland School of Population Health). The burden of cardiovascular disease in Australia for the year 2003. 2006.

[2] Balady GJ, Williams MA, Ades PA, Bittner V, Comoss P, Foody JM, Franklin B, Sanderson B, Southard D. Core components of cardiac rehabilitation/secondary prevention programs: 2007 update: a scientific statement from the American Heart Association Exercise, Cardiac Rehabilitation, and Prevention Committee, the Council on Clinical Cardiology; the Councils on Cardiovascular Nursing, Epidemiology and Prevention, and Nutrition, Physical Activity, and Metabolism; and the American Association of Cardiovascular and Pulmonary Rehabilitation. Circulation. 2007;115(20):2675-2682

[3] R.J. Thomas, "Cardiac Rehabilitation/Secondary Prevention Programs; A Raft for the Rapids: Why Have We Missed The Boat?", Circulation 2007;116:1644-1646.

[4] Scott IA, Lindsay KA, Harden HE. Utilisation of outpatient cardiac rehabilitation in Queensland. Med J Aust. 2003;179(7):332-333.

[5] Cooper AF. Factors associated with cardiac rehabilitation attendance: a systematic review of the literature. Clin Rehabil. 2002;16(5):541-552.

[6] RF. DeBusk, MULTIFIT: a new approach to risk factor modification. Cardiol Clin. 1996;14(1):143-157.

[7] K. Jolly, et.al., 2008 The Birmingham Rehabilitation Uptake Maximisation Study (BRUM): a randomised controlled trial comparing home-based with centre-based cardiac rehabilitation., Heart

[8] http://www.theheartmanual.com/aboutthemanual/default.htm

[9] Arrigo, I., Brunner-Larocca, H., Lefkovits, M.; Pfisterer, M. \& Hoffmann, A. (2008), Comparative outcome one year after formal cardiac rehabilitation: the effects of a randomized intervention to improve exercise adherence., Eur J Cardiovasc Prev Rehabil 15(306310)

[10] Ahtinen A., L. Hynninen, E. Koskinen, E. Mattila, J. Salminen, A. Väätäinen, "User Experiences of Mobile Wellness Applications in Health Promotion", Pervasive Health 09, London, April 2009.

[11] G. Lee, C. Tsai, W. G. Griswold, F. Raab, and K. Patrick, PmEB: a mobile phone application for monitoring caloric balance, in Proc. Conf. Human Factors in Computing Systems (CHI 2006), 2006, pp. 10131018.

[12] Mattila E, Pärkkä J, Hermersdorf M, Kaasinen J, Vainio J, Samposalo K, Merilahti J, Kolari J, Kulju M, Lappalainen R, Korhonen I. Mobile diary for wellness management - results on usage and usability in two user studies. IEEE T-ITB 2008;12(4):501-512.

[13] S. Consolvo, P. Klasnja, D. W. McDonald, D. Avrahami1, J. Froehlich, L. LeGrand, R. Libby, K. Mosher, J. A. Landay, Flowers or a Robot Army? Encouraging Awareness \& Activity with Personal, Mobile Displays, UbiComp'08, September 21-24, 2008, Seoul, Korea.

[14] Recommended Framework for Cardiac Rehabilitation '04, National Heart Foundation of Australia, ed; 2004

[15] http://www.nokia.com/betalabs/wellnessdiary

[16] http://www.nokia.com/betalabs/stepcounter

[17] E. Koskinen, J. Salminen,A Customizable Mobile Tool for Supporting Health Behavior Interventions, IEEE EMBC 07, Lyon, France, August 2007.

[18] E. Lamminmäki, J. Pärkkä, M. Hermersdorf, J. Kaasinen, K. Samposalo, J. Vainio, J. Kolari, M. Kulju, R. Lappalainen, I. Korhonen, "Wellness Diary for Mobile Phones." In Proc. EMBEC ‘05.

[19] E. Mattila, J. Koskelo, R. Lappalainen, J. Salminen, P. Nyman, J. Lähteenmäki, T. Leino, I. Korhonen, "Personal Health and Wellness Management: Citizen Empowerment through Mobile Technologies", IEEE EMBC 07, Lyon, France, August 2007.

[20] http:/sportstracker.nokia.com

[21] Reducing Risk in Heart Disease 2007, Guidelines for preventing cardiocvascular events in people with coronary heart disease, National Heart Foundation of Australia, ed; 2007.

[22] openid.net

[23] http://www.multi-ed.com/index.html

[24] http://bluetooth.com/Bluetooth/Products/Low_Energy.htm

[25] http://www.continuaalliance.org/home 